\title{
Medical marijuana research for chronic pain
}

\author{
Dermot P Maher, Steven P Cohen \\ Department of Anesthesiology and Critical Care Medicine, Pain Medicine Division, Johns Hopkins \\ Hospital, Baltimore, MD 21205, USA
}

Cannabinoids have been used for millennia to alleviate suffering. According to the sacred Hindu texts the Vedas, cannabis is a divine plant. Notwithstanding its long history in healing, cultural, and recreational contexts, few medical treatments are as polarising as medical marijuana (MMJ). ${ }^{1}$ Opinions reside along a spectrum ranging from a panacea that can alleviate all suffering to a hardcore drug with little clinical utility that is being medicalised in a veiled attempt to legitimise recreational use. ${ }^{1}$ Similar to other hot-button topics, viewpoints are influenced by cultural prejudices.

Chronic pain and psychiatric morbidity considerably overlap, including depression, substance abuse, anxiety disorders, post-traumatic stress, somatisation, and catastrophisation. ${ }^{2}$ This relationship is a two-way street as poorly controlled psychiatric conditions might not only be a consequence of chronic pain, but these conditions might also predispose individuals to develop chronic pain. Ironically, MMJ is often touted as an effective treatment for many of these psychiatric conditions. ${ }^{3}$

Evidence is mounting to support MMJ to treat chronic pain, in particular neuropathic and spasticity-related pain. ${ }^{2,4}$ Yet, despite some high-profile trial failures, MMJ therapy continues to generate attention from both the medical community and lay public. ${ }^{5}$ The literature does not provide adequate guidance for identification of phenotypes or pain conditions likely to benefit from MMJ. For any treatment associated with substantial sideeffects, including cannabinoids, identification of likely responders is paramount to establishment of a balanced approach. The evidence gap leaves physicians and patients reliant on low-quality studies to guide care. Similar to other treatments, large-scale studies devoid of bias should be done, and regulatory protocols and standards adhered to so informed treatment decisions can be made.

Although US Federal regulatory agencies ostensibly support an increase in clinical research into MMJ, they are constrained by laws that make possession of marijuana a federal crime. ${ }^{6}$ The Food and Drug Administration's position is that MMJ research conducted in states with enacted MMJ legislation should adhere to federal and scientific standards. The Food and Drug Administration has also supported state-level right-to-try bills, which allow patients access to medications still under investigation, but they will not change the status of MMJ to schedule 2 , which designates a drug as having medicinal uses but high abuse potential. ${ }^{7}$

dmaher3@jhmi.edu.

SPC is a member of the Advisory Board of Zynerba Pharmaceuticals. DPM declares no competing interests. 
Eight US states and the District of Columbia allow for recreational use of marijuana, whereas 28 states and the District of Colombia have some form of MMJ legislation in place. All states where recreational use is permitted, underwent a transition period whereby only medical use was allowed. The legislation in each of the four states that voted in favour of MMJ's use in the 2016 US election allows certification of medical necessity for conditions not otherwise specified. This legislation allows for MMJ's potential use in conditions not rigorously investigated and in those for which evidence for its use has been largely refuted in clinical trials, such as glaucoma. ${ }^{2}$ This legislation could also signify aggressive involvement in the legislative process by parties with financial interests and those desiring recreational approval. For state certification guidelines to fall in line with peer-reviewed medical literature would be more ethically and scientifically justifiable than would the current legislation. Lax indications might result in a larger marketplace than might stricter indications and increased tax revenue, but will ultimately lead to mistrust in physicians, increase the risk-to-benefit ratio (similar to what transpired for opioid treatment for noncancer pain), and predispose a promising treatment to premature failure.

To think medical and recreational use of marijuana do not considerably overlap would be naive, and we should avoid taking the same path we took with opioids. Among inpatients with psychiatric disorders and the ability to possess MMJ in Colorado, $41.4 \%$ admitted having sold or given away the drug to individuals without prescriptions, which is similar to the diversion proportion in other countries. ${ }^{8}$ Although sure to be controversial, state governments could allow both recreational and medical use, which would reduce the proportion of the drug diverted.

Multiple risk stratification tools are available for doctors to use when assessing patients for opioid use, but none have been validated for MMJ. The pharmacokinetics of MMJ more closely resemble those of opioids than of alcohol in that blood concentrations and psychomotor effects are weakly associated, which could have both regulatory (eg, compliance monitoring) and legal (eg, ascertainment of impairment) ramifications. Unfortunately, MMJ is also similar to opioids in that robust data establishing long-term benefit for chronic pain are scarce.

Chronic pain is an international epidemic with no simple solution. Whereas cannabinoid signalling pathways present intriguing possibilities to develop novel tools for management of chronic pain, previous to widespread acceptance, its role should be clearly delineated through standard regulatory processes, similar to any other new treatment. Increased research, coupled with growing public demand for alternative pain treatments, will drive further understanding of the role MMJ plays in pain management, allowing for development of responsible legislation devoid of bias.

\section{References}

1. Annas GJ. Medical marijuana, physicians, and state law. N Engl J Med 2014; 371: 983-85. [PubMed: 25099473]

2. Hill KP. Medical marijuana for treatment of chronic pain and other medical and psychiatric problems: a clinical review. JAMA 2015; 313: 2474-83. [PubMed: 26103031] 
3. Stapinski LA, Montgomery AA, Araya R. Anxiety, depression and risk of cannabis use: examining the internalising pathway to use among Chilean adolescents. Drug Alcohol Depend 2016; 166: 10915. [PubMed: 27427415]

4. Andreae MH, Carter GM, Shaparin N, et al. Inhaled cannabis for chronic neuropathic pain: a metaanalysis of individual patient data. J Pain 2015; 16: 1221-32. [PubMed: 26362106]

5. GW Pharmaceuticals and Otsuka announce results from two remaining sativex phase 3 cancer pain trials. 2015 http://www.fiercepharma.com/pharma/gw-pharmaceuticals-and-otsuka-announceresults-first-of-three-sativex-r-phase-3-cancer-pain (accessed Dec 20, 2015).

6. Savage SR, Romero-Sandoval A, Schatman M, et al. Cannabis in pain treatment: clinical and research considerations. J Pain 2016; 17: 654-68. [PubMed: 26961090]

7. FDA and marijuana: questions and answers. 2016 https://www.fda.gov/newsevents/ publichealthfocus/ucm421168.htm (accessed Oct 6, 2016).

8. Nussbaum AM, Thurstone C, McGarry L, Walker B, Sabel AL. Use and diversion of medical marijuana among adults admitted to inpatient psychiatry. Am J Drug Alcohol Abuse 2015; 41: 16672. [PubMed: 25375878] 